\title{
Increased numbers of pulmonary megakaryocytes in patients with arterial pulmonary tumour embolism and with lung metastases seen at
} necropsy

\author{
F A Soares
}

\begin{abstract}
Aims: To compare the number of pulmonary megakaryocytes in patients with local malignant disease without metastases with the numbers in patients with pulmonary tumour emboli without lung metastases and with those with pulmonary metastases.
\end{abstract}

Methods: The prevalence of pulmonary megakaryocytes was studied in 40 necropsies divided into four groups of 10 cases each: normal lungs (I); localised malignancies (II); pulmonary tumour embolism without lung metastases (III); pulmonary tumour embolism and lung metastases (IV). Five fragments (one of each pulmonary lobe) of tissue lung were collected, embedded in paraffin wax, sectioned, and stained by an immunohistochemical method to detect factor VIII related antigen. The number of megakaryocytes was evaluated in 500 high power fields/case.

Results: No differences were observed between groups I and II or between groups III and IV, but there was a 3.5-fold increase in the number of megakaryocytes in the groups with pulmonary tumour embolism or lung metastases compared with those with local neoplasms or normal lungs.

Conclusions: An increased number of pulmonary megakaryocytes correlated with the presence of tumour cells in the microcirculation of the lungs or parenchymal metastases, but not with local malignancies without lung disease. The permanent siting of tumour emboli may stimulate megakaryocytes to migrate to the lungs, and may increase the release of platelets into the pulmonary circulation.

Department of Pathology, Faculty of Medicine of Ribeirão Preto, University of São Paulo, Brazil F A Soares

Correspondence to: Dr Fernando Augusto Soares, Department of Pathology, HSC-2V17, McMaster University, 1200 Main Street West, Hamilton, Ontario, Canada L8N $3 Z 5$

Accepted for publication 27 August 1991
Megakaryocytes within pulmonary alveolar capillaries were first described by Aschoff in patients with pneumonia. ${ }^{1}$ After the bone marrow and the spleen, the lungs act as the third major reservoir for megakaryocytes. They are found in increased numbers in certain diseases such as acute infection, adult respiratory distress syndrome, atherosclerosis, thromboembolic disease, intravascular coagulation, myocardial infarction, liver failure, shock, and respiratory diseases. ${ }^{2-4}$ There is a positive correlation with the increased number of pulmonary megakaryocytes in malignant disease,${ }^{2}$ but this has been questioned by other authors. ${ }^{4}$ Platelet consumption and consequent thrombopoiesis are the main hypotheses for this increase in the numbers of pulmonary megakaryocytes. ${ }^{34}$

The objective of this study was to determine whether pulmonary megakaryocytes increase in patients with local malignancies without metastases, and to compare the findings with those obtained from patients with tumour emboli in the lungs but without parenchymal metastases, and with those with cancer and pulmonary metastases.

\section{Methods}

Pulmonary megakaryocytes were studied prospectively in 40 necropsies performed between 1986 and 1990 in the Department of Pathology, Faculty of Medicine of Ribeirão Preto, University of São Paulo, Brazil (figs 1 and 2). There was no evidence of peripheral venous thrombosis or signs of disseminated intravascular coagulation in any of the cases studied. The cases were divided into four groups.

Group I: Ten patients with normal lungs on gross and histological examination and without any condition known to be associated with increased numbers of pulmonary megakaryocytes. The group comprised five cases of epilepsy, three of tetanus, and two of brain trauma. Rib fractures or other associated disease were absent.

Group II: Ten patients with localised malignancies without metastatic lung disease. No distant metastases were observed at necropsy and the primary sites of the neoplasms were as follows: three cases of adenocarcinoma of the stomach, two cases of adenocarcinoma of the papilla of Vater, two cases of squamous cell carcinoma of the larynx, one hepatocarcinoma, one adenocarcinoma of the rectum and one renal cell carcinoma.

Group III: Ten patients with malignancies without pulmonary metastases but with arterial tumour embolism to the lungs. Pulmonary tumour embolism was defined as isolated or grouped tumour cells found within arteries or arterioles. The primary sites of the cases were as follows: three cases of adenocarcinoma of the pancreas, three cases of hepatocarcinoma, two cases of adenocarcinoma of the stomach, one case of renal cell carcinoma and one squamous cell carcinoma of the vulva.

Group IV: Ten patients with malignancies with arterial tumour embolism and pulmonary parenchymal metastases. The primary sites 


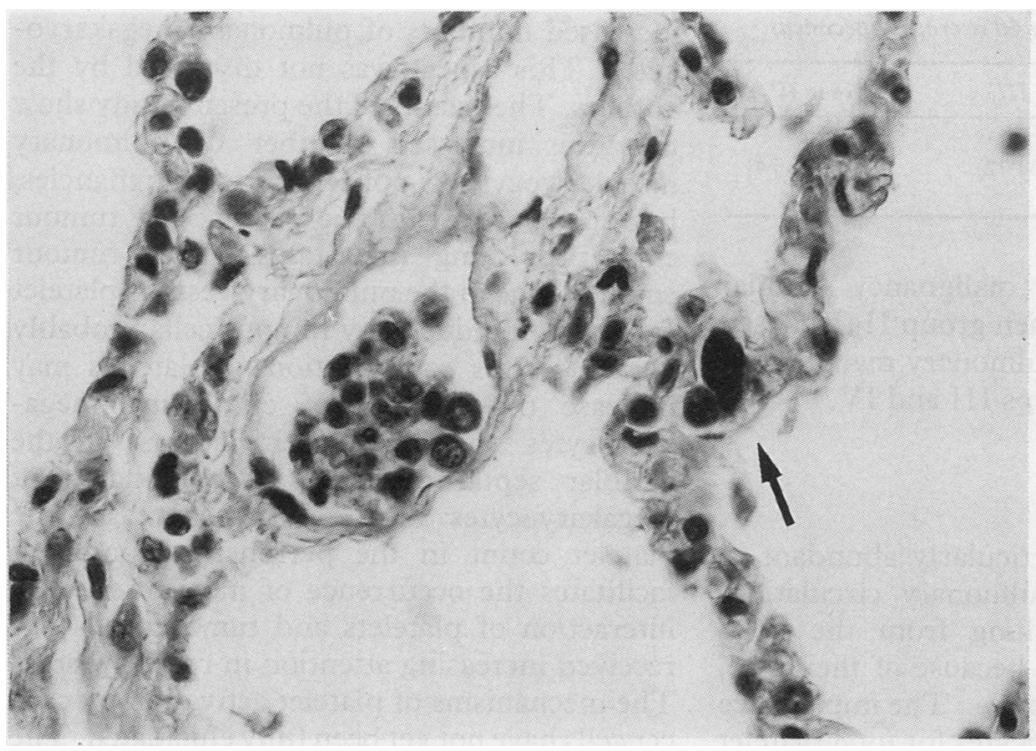

Figure 1 Megakaryocytes in the alveolar septal capillaries (arrow) in a case of pulmonary tumour embolism. Note the tumour emboli in an arteriole (haematoxylin and eosin).

of the cases were as follows: three cases of adenocarcinoma of the stomach, two cases of adenocarcinoma of the pancreas, two cases of hepatocarcinomas, one case of squamous cell carcinoma of the oesophagus, one of renal cell carcinoma and one of squamous cell carcinoma of the larynx.

The lungs were removed as a block and injected with formaldehyde through the trachea. After fixation five fragments of lung tissue were collected, one from each pulmonary lobe from the middle portion between the hilum and pleural surface. ${ }^{3-5}$ The tissues were embedded in paraffin wax for routine histological processing, cut into $5 \mu \mathrm{m}$ sections, and stained with haematoxylin and eosin. Immunohistochemistry was carried out using antiserum against factor VIII related antigen (F VIII RAg; DAKO) to separate megakaryocytes from neoplastic cells. The antiserum was applied to sections at a 1 in 200 dilution after

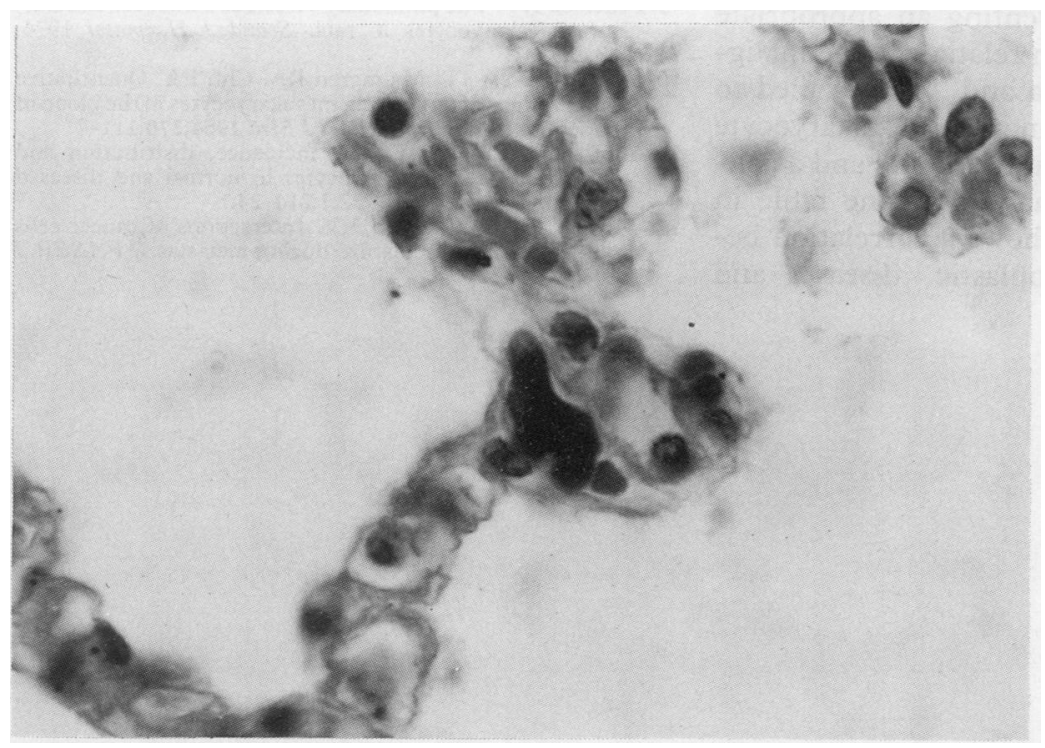

Figure 2 Detail of a megakaryocyte in the alveolar septal capillary (haematoxylin and eosin). digestion with trypsin and after blocking the endogenous peroxidase with $0.3 \%$ hydrogen peroxide in methanol. We applied the peroxidase-antiperoxidase staining procedure as described by Crocker and Smith. ${ }^{6}$

One hundred high power fields from each section were examined histologically, for a total of 500 high power fields. Megakaryocyte screening was performed using a Zeiss Jenamed 2 routine transmitted light microscope with a $\times 40$ objective and a $\times 10$ eyepiece. The limit of each field was determined by a line in the eyepieces which prevented repeated counts of the same field. Care was taken to avoid areas with major vessels, large airways, emphysema, atelectasis, metastasis or any other pathoanatomical changes.

Data were analysed statistically by the Kruskal-Wallis test, with the level of significance set at 0.05 .

\section{Results}

Figure 2 shows the pulmonary megakaryocyte counts for the four groups and their statistical correlations.

Megakaryocytes were found in the lungs of all 40 necropsy specimens studied (table).

Statistical analysis showed no differences between group I (normal lungs) and group II (localised neoplasms with no pulmonary metastases) or between group III (tumour embolism) and group IV (lung metastasis). When group I (normal lungs) was compared with groups III (tumour embolism) and IV (lung metastasis), statistically higher megakaryocyte counts were observed in the two

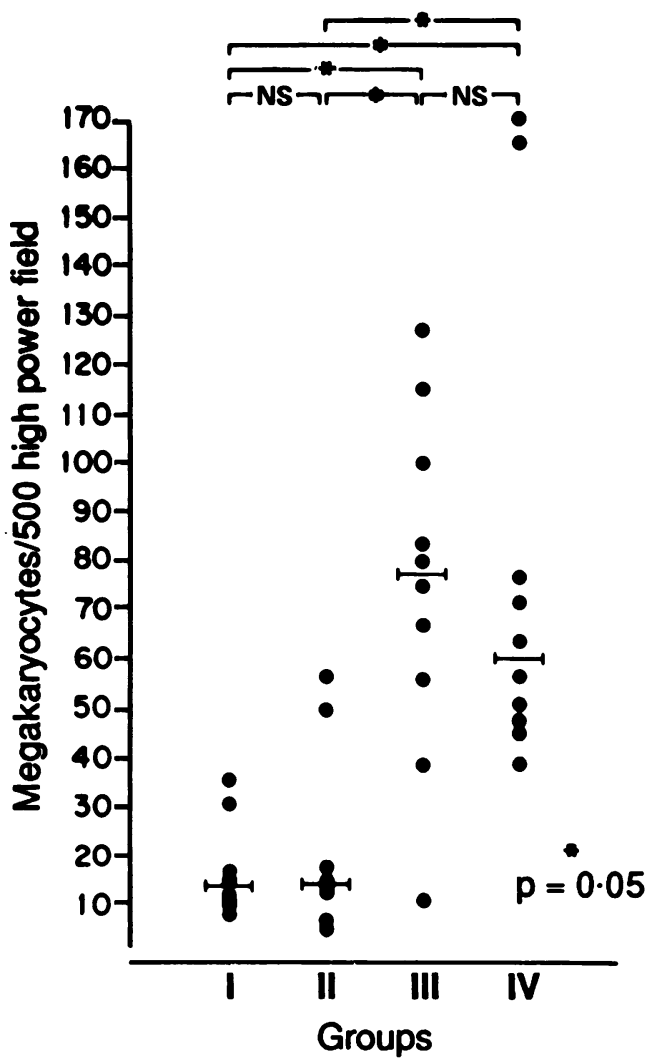

Figure 3 Pulmonary megakaryocyte counts in the 4 groups studied and their statistical correlation. NS = not significant; line $=$ median. 
Numbers of megakaryocytes per 500 high power fields found in 40 necropsy specimens

\begin{tabular}{lclll}
\hline & Group I & Group II & Group III & Group IV \\
\hline Range & $8-36$ & $5-57$ & $11-127$ & $39-170$ \\
Mean (SD) & $16 \cdot 8(9 \cdot 25)$ & $21 \cdot 5(17 \cdot 62)$ & $75 \cdot 4(34 \cdot 72)$ & $79 \cdot 7(48 \cdot 24)$ \\
Median & 14 & 14.5 & $77 \cdot 5$ & 60.5 \\
\hline
\end{tabular}

groups with pulmonary malignancy. Similar results were obtained when group II (localised malignancies with no pulmonary malignancy) was compared with groups III and IV.

\section{Discussion}

Megakaryocytes are particularly abundant in the lung and in the pulmonary circulation. They migrate to the lung from the bone marrow, and, probably, because of their size, remain in alveolar capillaries. ${ }^{7}$ The importance of pulmonary megakaryocytes for total platelet production is still controversial. ${ }^{89}$ The platelet count is higher in the pulmonary vein than in the pulmonary artery, suggesting that pulmonary megakaryocytes release platelets. ${ }^{49}$

Increased numbers of pulmonary megakaryocytes have been reported for various diseases, including malignancies. Hume et al showed that circulating megakaryocytes are more common in the peripheral and local blood of patients with malignancies than in controls, but the authors failed to show a relation with the extent of the cancer. ${ }^{10}$ Aabo and Hansen reported increased numbers of pulmonary megakaryocytes in $45 \%$ of the cases of cancer, with a significant difference when compared with the control group. ${ }^{3}$ The authors proposed consumption coagulopathy as a common pathogenic mechanism to explain the increased number of pulmonary megakaryocytes in malignancies and in other diseases. ${ }^{3}$ Sharnoff and Kim studied 37 cases of malignant disease and reported an intense increase in pulmonary megakaryocytes in 10 of them. ${ }^{2}$ Smith and Butgher included 14 malignant neoplasms in a "miscellaneous" group which consisted of 50 cases with different conditions such as myocardial infarct, peptic ulcer, congenital heart disease, etc., thus preventing an appropriate analysis of the results in relation to the malignant tumours. ${ }^{11}$ Sharma and Talbot failed to show differences in pulmonary megakaryocyte counts between malignant tumours and a control group. ${ }^{4}$ Careful analysis of one table in their study, however, shows a correlation between metastatic neoplastic disease and increased numbers of pulmonary megakaryocytes. This aspect was not discussed by the authors. The results of the present study show that the increased number of pulmonary megakaryocytes is not related to malignancies, but specifically with the presence of tumour emboli or lung metastases. After tumour emboli lodge in the pulmonary vessels, platelet aggregation induced by tumour cells probably occurs. ${ }^{12}$ This consumption of platelets may increase the number of circulating megakaryocytes and hence their increase in the alveolar septal capillaries. The lodging of megakaryocytes in the lungs increases the platelet count in the peripheral blood and facilitates the occurrence of metastases. The interaction of platelets and tumour cells has received increasing attention in recent years. ${ }^{12}$ The mechanisms of platelet activation by cancer cells have not yet been fully elucidated. The release of vasoconstrictive products, adhesion glycoproteins, and platelet membranes may themselves promote tumour cell aggregating activity and facilitate the spread of metastases. ${ }^{12}$

We are grateful to Mr JL de Souza and Mrs AMA Abreu for technical assistance, and to Dr Gerson Muccilo for statistical assistance.

This work was supported by grants from FAPESP (No 90/34408) and FAEPA.

1 Aschoff L. Über capilläre Embolie von Riesenkernzellen. Virchows Arch (Path Anat) 1893;134:11-24.

2 Sharnoff JG, Kim ES. Evaluation of pulmonary megakaryocytes. JAMA 1958;66:176-82.

3 Aabo K, Hansen KB. Megakaryocytes in pulmonary blood vessels. 1. Incidences at autopsy, clinicopathological relations especially to disseminated intravascular coagulation. Acta Pathol Microbiol Scandinavica (Sect A) 1978;86. 285-91.

4 Sharma GK, Talbot IC. Pulmonary megakaryocytes: "missing link" between cardiovascular and respiratory disease? J Clin Pathol 1986;39:969-76.

5 Bendic-Hansen K, Aabo K. Distribution of intrapulmonary megakaryocytes. Acta Pathol Microbiol Scandinavica megakaryocytes. Acta Path

6 Crocker J, Smith PJ. Value of factor VIII related antigen as a means of demonstrating extramedullary megakaryopoiesis. J Clin Pathol 1984;34:843-5.

7 Pedersen NT. Occurrence of megakaryocytes in various vessels and their retention in the pulmonary capillaries in man. Scand J Haematol 1978;21:369-75.

8 Kaufman RM, Airo R, Pollack S, Crosby WH. Circulating megakaryocyte and platelet release in the lung. Blood 1965;26:720-9.

9 Pedersen NT. The pulmonary vessels as a filter for circulating megakaryocytes in rats. Scand J Haematol 1974; ing megak

10 Hume R, West JT, Malmgren RA, Chu EA. Quantitative observations of circulating megakaryocytes in the blood of patients with cancer. $N$ Engl J Med 1964;270:111-7.

11 Smith EB, Butgher J. The incidence, distribution and significance of megakaryocytes in normal and diseased human tissues. Blood 1952;7:214-24.

12 Weiss L, Orr FW, Honn KV. Interactions of cancer cells with the microvasculature during metastasis. FASEB J 1988;2:12-21. 